\title{
En bloc pelvic resection of ovarian cancer with rectosigmoid colectomy: a literature review
}

\author{
Myeong-Seon $\mathrm{Kim}^{1}$, Joseph J. Noh², Yoo-Young Lee ${ }^{2}$ \\ ${ }^{1}$ Division of Gynecologic Oncology, Department of Obstetrics and Gynecology, St. Vincent's Hospital, College of Medicine, The Catholic University \\ of Korea, Seoul, Korea; ${ }^{2}$ Division of Gynecologic Oncology, Department of Obstetrics and Gynecology, Samsung Medical Center, Sungkyunkwan \\ University School of Medicine, Seoul, Korea \\ Contributions: (I) Conception and design: YY Lee; (II) Administrative support: JJ Noh; (III) Provision of study materials or patients: MS Kim; (IV) \\ Collection and assembly of data: MS Kim; (V) Data analysis and interpretation: MS Kim; (VI) Manuscript writing: All authors; (VII) Final approval \\ of manuscript: All authors. \\ Correspondence to: Yoo-Young Lee, MD, PhD. Clinical Professor, Division of Gynecologic Oncology, Department of Obstetrics and Gynecology, \\ Samsung Medical Center, Sungkyunkwan University School of Medicine, 81, Irwon-ro, Gangnam-gu, Seoul 06351, Korea. \\ Email: yooyoung.lee@samsung.com.
}

\begin{abstract}
Maximal cytoreductive surgery is an important prognostic factor in advanced epithelial ovarian cancer (EOC). To achieve maximal cytoreductive surgery, en bloc pelvic resection with rectosigmoid colectomy can be an effective surgical strategy. This surgical methodology was first described in 1968 as "radical oophorectomy." Since then, it has been adopted by many medical institutions around the world, and its safety has been shown by many studies. However, research on the surgical method is still lacking due to the limited number of prospective comparative studies. We will review the journals on en bloc pelvic resection with rectosigmoid colectomy published to date and discuss its efficacy, complications, and surgical techniques of the procedures.
\end{abstract}

Keywords: Ovarian cancer; ovariectomy/methods; ovarian neoplasms/surgery; cytoreductive surgery; colectomy; en bloc pelvic resection with rectosigmoid colectomy; radical oophorectomy; optimal debulking

Submitted Dec 31, 2019. Accepted for publication Apr 28, 2020.

doi: 10.21037 /gs-19-540

View this article at: http://dx.doi.org/10.21037/gs-19-540

\section{Introduction}

\section{Role of maximal cytoreductive surgery in ovarian cancer}

Maximal cytoreductive surgery followed by platinum-based adjuvant chemotherapy is considered the most effective therapeutic strategy in the treatment of advanced epithelial ovarian cancer (EOC) to date $(1,2)$. Patient survival has been shown to be negatively correlated with residual tumor diameter in many studies, emphasizing the role of maximal cytoreductive surgery. The favorable prognostic effect of maximal cytoreduction on advanced EOC has been investigated since it was first reported in 1975 by Griffiths (3-10). Griffiths showed that patient survival improved as residual tumor size decreased in 102 patients with FIGO (Fédération Internationale de Gynécologie et d'Obstétrique) stage II-
III EOC. Bertelsen also reported significant differences in five-year survival rates between the patients who received optimal debulking surgery and those who received suboptimal surgery in advanced EOC ( $46 \%$ vs. $15 \%, \mathrm{P}<0.001)$. These differences were also shown in the disease progression rates (10\% in optimal surgery group vs. $43 \%$ in suboptimal surgery group, $\mathrm{P}<0.001)(11)$. In another study in which Bristow et al. performed meta-analysis of 81 cohorts of patients with advanced EOC (6,885 patients), the authors found each $10 \%$ increase in maximal cytoreduction was associated with a $5.5 \%$ increase in median survival time (10).

\section{Role of rectosigmoid colectomy to achieve optimal debulking}

Rectosigmoid colectomy is an important part of the 
debulking procedures in advanced EOC. In a retrospective review in which the authors analyzed 527 patients who underwent primary cytoreductive surgery for FIGO stage II-IV EOC, 458 (79\%) patients were optimally cytoreduced (largest residual tumor diameter less than $2 \mathrm{~cm}$ ), and 144 $(27.3 \%)$ of these required bowel resections in order to accomplish this. Of the 144 patients who received bowel resections, 81 patients received rectosigmoid resection only (12). Other studies have also demonstrated that the most commonly involved bowel segment in surgeries for advanced EOC is the rectosigmoid colon $(12,13)$.

There are two surgical techniques for rectosigmoid colectomy - the total mesorectal excision (TME) and the close rectal dissection (CRD). The main difference between the two techniques is whether it preserves mesorectum and its vascular supply. The TME removes all mesorectal tissue, thereby reducing blood supply to its nearby bowels whereas the CRD technique preserves them. The TME technique has been associated with the reduction of local recurrence in colorectal cancer $(14,15)$. However, these results may not be directly applied to EOC, because EOC has a different biology and patterns of metastasis compared to colorectal cancer. EOC often spreads into the peritoneal cavity along the peritoneal surface and makes tumor deposits on the serosa of rectosigmoid colon first, then invades into the muscular layers followed by the mucosa, which is the opposite of the direction in colorectal cancer. In fact, studies have shown that there was no difference in pelvic recurrence or progression free survival (PFS) between the patients with advanced EOC who received rectosigmoid colectomy by the CRD technique versus the TME technique (16). They further showed that rectosigmoid colectomy by the CRD technique was associated with less anastomotic leakage and low incidence of prolonged ileus over 7 days in comparison with the TME technique, presumably due to the preserved blood supply (16). Since there are only limited studies comparing the TME versus the CRD in rectosigmoid colectomy in advanced EOC, further studies are needed.

Kim et al. investigated the survival impact of low anterior resection (LAR) and tumorectomy in patients with EOC whose tumors were grossly confined in the pelvis (17). The authors retrospectively reviewed and compared the outcomes between LAR and tumorectomy of 92 patients with rectosigmoid lesions. There were no significant differences between the two groups in PFS and overall survival (OS). However, postoperative ileus was more frequently observed in the patient treated with LAR than those with tumorectomy. The authors concluded that if tumor infiltration was up to the serosa and subserosa of the rectosigmoid colon, then rectosigmoid lesion could be resected completely via tumorectomy. In other words, LAR seemed to be not mandatory to improve prognosis in patients with EOC with gross tumors confined in the pelvis. However, due to limited data, further research may be needed to standardize the results of the aforementioned study to all patients with advanced EOC. Therefore, except when completely removed by tumorectomy, bowel surgery is an important part of the debulking procedures for optimal cytoreductive surgery.

\section{Concept of en bloc pelvic resection}

En bloc pelvic resection of ovarian cancer with rectosigmoid colectomy was first described in 1968 and 1973 independently by Hudson and Chir, which was termed "radical oophorectomy" $(18,19)$. Since then, it has been adopted by many medical institutions around the world and various terminologies have been developed to describe the procedures with additional modifications: en bloc rectosigmoid colectomy (20-24), reverse hysterocolposigmoidectomy (25), complete parietal and visceral peritonectomy (26), en bloc pelvic peritoneal resection of the intrapelvic viscera (27), and modified posterior exenteration (28).

In advanced EOC, pelvis and cul-de-sac are often obliterated due to tumor invasion. Therefore, it is not uncommon to confront indistinguishable anatomical structures of the pelvic organs during surgery. Tumor spreads to the peritoneal surfaces of bowel and bladder are common findings in advanced EOC. In such cases, en bloc pelvic resection of tumors with the affected nearby organs such as the uterus, pelvic peritoneum, or rectosigmoid colon is recommended. Considering the spread patterns of EOC and potential residues of microscopic lesions that may be invisible at the time of surgery, it may rather be advantageous for patients to undergo en bloc pelvic resections with rectosigmoid colectomy.

\section{Efficacy of en bloc pelvic resection with rectosigmoid colectomy}

Because EOC shows in many ways distinctly different disease characteristics from other solid neoplasms, it is difficult to perform randomized controlled trials (RCTs) to evaluate the efficacy of en bloc pelvic resections in patients with advanced EOC. Surgeons often make decisions in 
Table 1 Published journals of en bloc pelvic resection with rectosigmoid colectomy

\begin{tabular}{|c|c|c|c|c|c|c|}
\hline Author & Year & Patient, $\mathrm{n}$ & Stage & $\begin{array}{l}\text { Median HS, } \\
\text { days }\end{array}$ & Optimal rate, \% & Complication \\
\hline Bridges et al. (24) & $1984-1988$ & 43 & II-IV & 16 & $74.4^{\S}$ & Prolonged ileus (9.3\%) \\
\hline Obermair et al. (23) & $1996-2000$ & 65 & II-IV & 11 & 73.8 & $\begin{array}{l}\text { lleus }(7.7 \%) \text {; anastomotic leak (3.1\%); } \\
\text { fistula }(1.5 \%)\end{array}$ \\
\hline Clayton et al. (20) & 1989-2000 & 129 & I-IV & & 79.2 & $\begin{array}{l}\text { Prolonged ileus ( } 7.8 \%) \text {; anastomotic leak } \\
(0.8 \%) \text {; fistula }(1.6 \%)\end{array}$ \\
\hline Bristow et al. (31) & $1997-2001$ & 31 & III-IV & 11 & 87.1 & Anastomotic leak (3.2\%) \\
\hline Park et al. (30) & $2001-2005$ & $\begin{array}{l}60-\text { primary: } 46 \\
\text { recur: } 14\end{array}$ & $\begin{array}{l}\text { Primary: III-IV; } \\
\text { Recur: I-III }\end{array}$ & 18 & $\begin{array}{l}\text { Primary: } 97.9 \\
\text { Recur: } 78.6\end{array}$ & $\begin{array}{l}\text { Prolonged ileus: primary }(4.3 \%) \text {; } \\
\text { recur }(0.0 \%) \text {. Anastomotic leak: primary } \\
(2.1 \%) \text {; recur }(0.0 \%) \text {. Fistula: primary } \\
(2.1 \%) \text {; recur }(7.1 \%)\end{array}$ \\
\hline
\end{tabular}

$\S,<2 \mathrm{~cm}$ maximal size of residual tumor. HS, hospital stay; Recur, recurrent.

regards to the execution of particular surgical procedures intraoperatively after completion of thorough surgical exploration the disease status. Surgery for ovarian, fallopian, and peritoneal cancer is difficult to predict of surgical method before surgery, contributing reasons as to why it is often difficult to perform randomized controlled trials (RCT). The reported success rate of the scheduled en bloc pelvic resection was almost $100 \%$ in published journals $(20,23,24,29-31)$ (Table 1). In the United States, optimal resection is reported in about $70 \%$ of primary cytoreductive surgery in advanced EOC (32). Although it is difficult to make a direct comparison, in journals with en bloc surgery, the rate of optimal debulking is reported to be $73.8-100 \%(20,23,24,29-31)$. Bristow et al. reported $87.1 \%$ of optimal rate of type II-III radical oophorectomy in the advanced EOC with FIGO stage III-IV (31). Bridges et al. reported the retrospective surgical outcomes of 43 patients with advanced EOC treated with en bloc pelvic resection with concomitant rectosigmoid colectomy (24). Optimal cytoreduction was over $70 \%$ of the cases. There was no postoperative leakage of fistula. Sainz de la Cuesta et al. also reported the surgical outcomes of 30 patients who underwent en bloc resection of the pelvic peritoneum and all pelvic viscera with a rectosigmoid reanastomosis in advanced EOC (27). All patients underwent cytoreduction with residual disease less than $1 \mathrm{~cm}$. Andreas Obermair et al. reported safety and efficacy of low anterior en bloc resection with rectosigmoid resection followed by an end-to-end anastomosis for 65 consecutive patients with advanced EOC. No gross residual and residual tumors less than $1 \mathrm{~cm}$ was in observed in 14 patients (21.5\%) and 34 patients (52.3\%) respectively. Residual tumor size $1-2 \mathrm{~cm}$ and over $2 \mathrm{~cm}$ was in 10 patients $(15.4 \%)$ and 4 patients $(6.2 \%)$ respectively. Thirty-three patients had a diverting loop ileostomy and five patients had a colostomy. They reported that surgicalrelated complications such as wound complication, septicemia and anastomotic leakage were more frequent in patients with serum albumin level of $\leq 30 \mathrm{~g} / \mathrm{L}$ preoperatively. Clayton et al. reported a retrospective review of 129 consecutive patients who underwent en bloc pelvic resection with concomitant rectosigmoid colectomy (20). Optimal cytoreduction (residual tumor less than $2 \mathrm{~cm}$ ) was $79.2 \%$ and suboptimal cytoreduction was $18.5 \%$. Median survival of patients with optimal debulking was 37.6 months whereas patients with suboptimal debulking was 11.6 months. Park et al. reported a retrospective review of en $b l o c$ resection of 46 primary and 14 recurrent EOC patients who underwent en bloc resection (30). For primary advanced EOC patients, no residual tumor and optimal cytoreduction (residual tumor less than $5 \mathrm{~mm}$ ) were $43.5 \%$ and $89.5 \%$, respectively. For recurrent patients, no visible tumor at the completion of surgery was $42.9 \%$ and optimal cytoreduction was achieved in $64.3 \%$. The patients with no residual tumor showed longer disease-free survival than those with residual tumor (median survival, 30 vs. 7 months, $\mathrm{P}=0.0082$ ) in primary advanced EOC. For recurrent patients, patients 
with no visible tumors also had longer disease-free survival than those with residual tumor (median survival, not reached vs. 5 months, $\mathrm{P}=0.0156)$. Chi et al. reported that surgical trends moving towards retroperitoneal en bloc pelvic resection including rectosigmoid colectomy in the mid1990 s led to a $40 \%$ increase in surgical radicality and at least $10 \%$ improve of median OS (33).

\section{Preoperative and postoperative managements of the rectosigmoid colectomy}

\section{Bowel preparation and prophylactic antibiotics}

The necessity of mechanical bowel preparation (MBP) in patients undergoing colectomy has been controversial. Recent meta-analyses demonstrated benefits of oral antibiotic bowel preparation (OABP) but it also showed that performing MBP only did not provide any benefits (34-36). It was also reported that MBP did not significantly reduce postoperative complications in elective colorectal surgery $(36,37)$. Moreover, it has been shown that patients who received MBP prior to surgery had a higher incidence of bowel content spillage, which might increase the rates of postoperative complications (38). In another study in which the authors investigated postoperative complications in elderly patients who were 75 years or older undergoing elective colectomy, both $\mathrm{OABP}$ and $\mathrm{OABP}$ plus MBP were associated with reduced morbidity compared with no bowel preparation (38). Even in the absence of MBP, OABP seemed to reduce the incidence of surgical site infection (SSI) following elective colectomy (39). The enhanced recovery after surgery (ERAS) protocols recommend to avoid routine MBP in colonic surgery due to the concerns of dehydration, distress to patients, prolonged ileus, and lack of efficacy $(40,41)$.

In the Cochrane review, the authors also found high quality evidence that antibiotics covering aerobic and anaerobic bacteria delivered orally or intravenously prior to elective colorectal surgery adequately reduced the risk of surgical wound infection by as much as $75 \%$ (42).

\section{Fluid management}

Fluid management plays key roles in achieving optimal recovery after bowel surgery. Intravascular volume is the key elements of cardiac output that deliver oxygen to the peripheral tissues. Intravascular hypovolemia especially after surgery can lead to hypoperfusion of organs including the bowel and it can lead to the disturbance of the healing process, subsequently resulting in postoperative complications (41). Preoperative dehydration should be minimized if possible. Studies have shown that the intake of clear fluid up to 2 hours prior to the induction anesthesia did not increase surgical morbidity (40). Postoperative fluids should be administrated minimally to maintain normovolemia and be careful of fluid excess. Balanced crystalloid such as $0.9 \%$ saline is recommended as a fluid management after bowel surgery (41).

\section{Oral feeding}

Studies have consistently showed that early feeding after surgery was associated with significantly faster recovery of bowel function with shorter times to flatus and to tolerance of regular diet (43). The RCTs for early enteral or oral feeding also showed that it reduced the risk of postoperative infection and that it was not associated with an increased risk of anastomotic leakage or dehiscence (44-46). Based on the results of these studies, early feeding practices have been adopted widely and numerous professional societies advocate it.

\section{Complications of en bloc pelvic resection with rectosigmoid colectomy}

None of the studies published to date have reported major mortalities directly associated with en bloc pelvic resection with rectosigmoid colectomy. Anastomotic leakage is considered the major complication of the surgical procedures so far. Some minor complications reported are prolonged ileus longer than 7 days, blood transfusion during or after operation, and the formation of fistula with nearby organs (47). Although it may be difficult to predict the risk of developing complications attributable to the surgical procedures beforehand, researchers have associated the levels of preoperative serum albumin less than $30 \mathrm{~g} / \mathrm{L}$ with anastomotic leakage, wound complications and septicemia (23).

\section{Anastomotic leakage}

Concerns remain about anastomotic leakage or dehiscence in patients undergoing en bloc pelvic resections with rectosigmoid colectomy. Patients with advanced EOC often have large volumes of ascites and are nutritionally compromised due to extensive disease burden and perhaps 
bowel obstruction accompanied with it. Such patients may undergo transient or permanent intestinal diversion such as colostomy or ileostomy to alleviate the symptoms. However, intestinal diversion does not seem to be superior to en bloc resections with rectosigmoid colectomy in regards to the rates of developing anastomotic leakage. Studies have reported 0 to $8 \%$ of incidence of anastomotic leakage among the patients who underwent rectosigmoid colectomy (20,22-25). Although these figures may increase to some extent when patients have large volumes of ascites and disease burden, it still seems to remain acceptably low. Researchers have reported $2.1 \%$ to $3.1 \%$ of incidence of anastomotic leakage in EOC patients with ascites greater than $500 \mathrm{~mL}$ at the time of the surgery $(23,28)$. From these data, prophylactic intestinal diversion is not required and anastomosis by using the staples and handsewing is considered as safe for rectosigmoid colectomy for advanced EOC. It is in general consensus that en bloc pelvic resections with rectosigmoid colectomy permits a high rate of complete debulking with acceptable morbidity and mortality rates.

The incidence of anastomotic leakage in en bloc pelvic resection with rectosigmoid resection seems to be no greater than that of general colectomy.

\section{Prolonged ileus after surgery}

Although variations exist in terms of the definition of the duration, it is generally considered that no return of bowel function in 7 days after surgery indicates that the patients suffer postoperative ileus. Postoperative ileus is one of the most common complications following colorectal surgery. The most commonly used indices are delayed return of flatus and/or defecation (90\%), intolerance of diet (63\%), reinsertion of a nasogastric tube $(20 \%)$, radiologic measures of bowel transit (11\%), and delayed return of bowel sounds (7\%) (22). It has been reported that male gender, advanced age, and major blood loss during operations are associated with high risk of developing postoperative ileus $(48,49)$. Despite diverse measures to prevent postoperative ileus such as early ambulation and feeding, $10-30 \%$ of patients are reported to develop postoperative ileus after colorectal surgery (50-56). The incidence of prolonged ileus in patients who underwent en bloc resection with rectosigmoid colectomy for advanced EOC has been reported to be 4.3$9.3 \%$, which is similar to that in general colorectal surgery $(19,22-24,36)$. It is well known that adequate perioperative nutritional status is important to prevent of postoperative ileus, along with short limitation of preoperative fasting to 6 hours for solid food and 2 hours for liquids. Coffee and chewing gum are also known to have preventive effect for postoperative ileus (57). With the utilization of these measures to prevent postoperative ileus, en bloc pelvic resections with rectosigmoid colectomy can be safely performed in patients with advanced EOC.

\section{Procedures of radical oophorectomy}

\section{Classification of radical oophorectomy}

To define the scope of surgical resection with uniform terminology, the classification of radical oophorectomy has been applied (31).

Type I radical oophorectomy: retrograde modified radical hysterectomy with en bloc resection of the adnexa, pelvic cul-de-sac, and involved pelvic peritoneum.

Type II radical oophorectomy: broadened to include an en bloc resection of the rectosigmoid colon below the peritoneal reflection with complete parietal and visceral pelvic peritonectomy.

Type III radical oophorectomy: an extension of the type I or II procedure incorporating a portion of urinary bladder and/or pelvic ureter.

Of these classifications, type II radical oophorectomy has been reported to be the most used: type I (18\%), type II (74\%), type III (8\%) (58).

\section{Surgical procedure of type II radical oophorectomy}

The radical oophorectomy is initiated by incising the paracolic gutters bilaterally. The paracolic gutter incisions are extended caudally, along the psoas muscles, to the posterior margin of the symphysis pubis (Figure 1).

Identifying the infundibulopelvic (IP) ligaments and round ligaments bilaterally, then suture ligatures bilaterally. Extensive tumor infiltration of the pelvis can obscure the round ligaments, in which case they can be located retroperitoneally, ligated, and divided as laterally as possible.

The pelvic dissection proceeds in a centripetal fashion. The pararectal and paravesical spaces are developed identifying the uterine artery and the cardinal ligament. The uterine vascular pedicles are skeletonized, doubly ligated, and divided at the level of the ureter bilaterally (Figure 2).

The ureters are identified within the pararectal space and mobilized from their attachments of the medial side of 

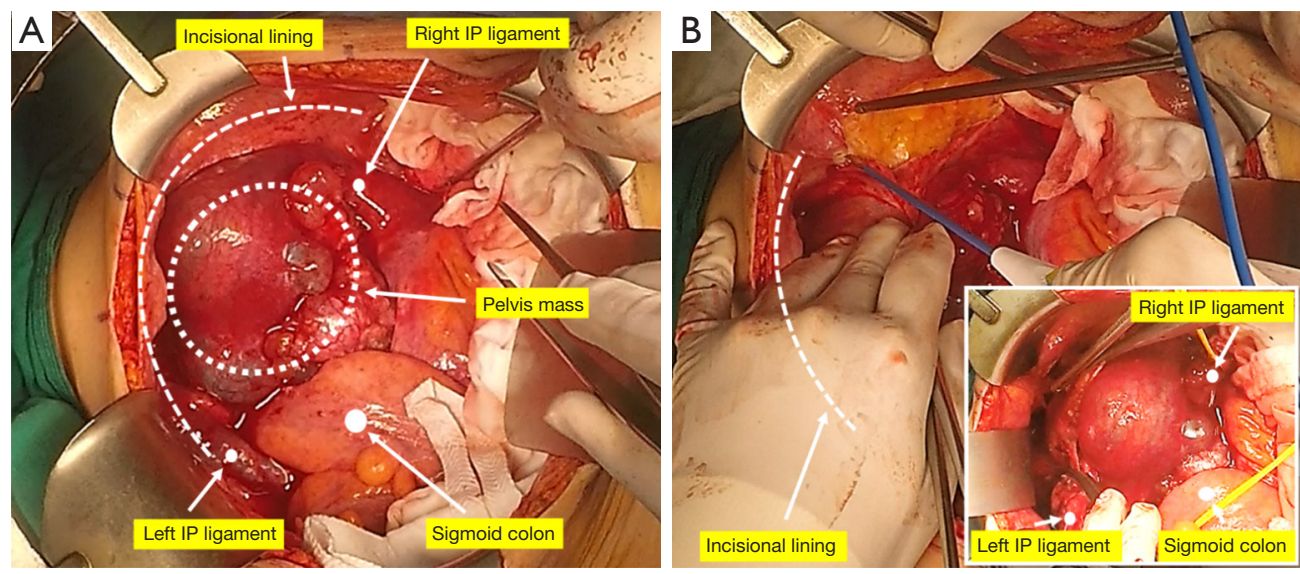

Figure 1 Radical oophorectomy initiated by the paracolic gutter incisions. (A) Pelvic mass (circle with white dots) causes adhesion of the organs of pelvis to obliteration. Sigmoid colon (bigger white dot) does not move due to adhesion of the pelvic mass. (B) Anterior pelvic peritonectomy. The paracolic gutter incisions are extended caudally to the anterior pelvic peritoneum. The bladder is placed in traction and the peritoneum of anterior pelvis is deperitonealized by using the electrosurgical unit.
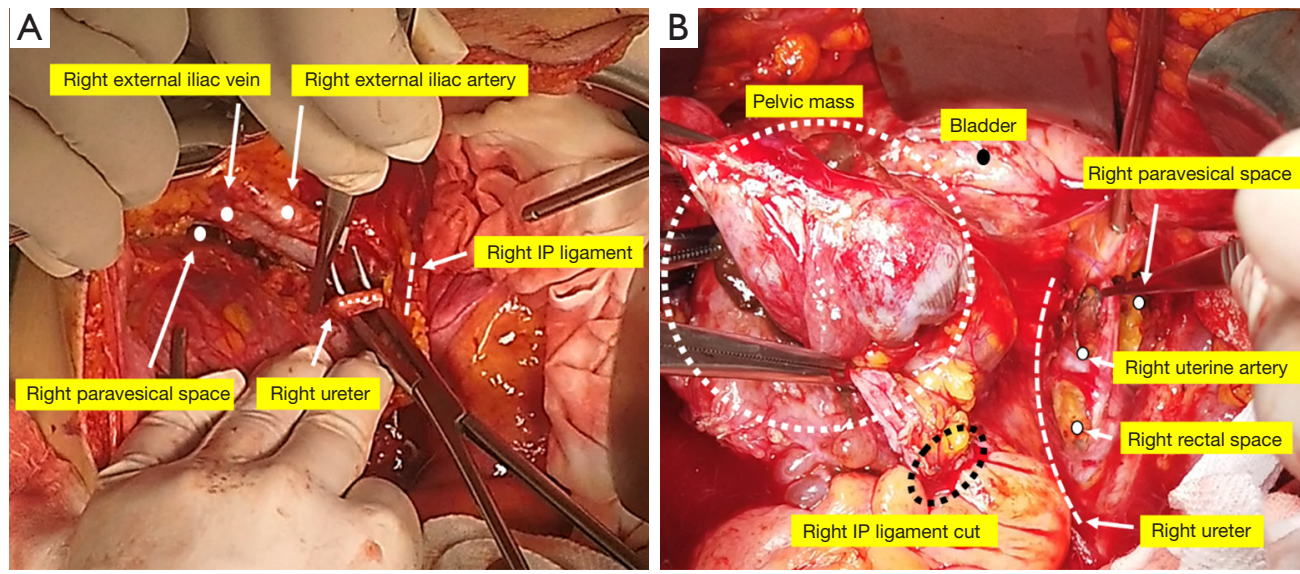

Figure 2 The pelvic dissection. (A) Identifying retropelvic space structure. The ureter is identified and mobilized from their attachment of the medial side of the pelvic peritoneum. (B) By suture ligatures of the IP ligament early in the course of operation, the pelvic mass is devascularized.

the pelvic peritoneum, coursing from the pelvic brim to the tunnel of Wertheim (Figure 3).

The anterior pelvic peritoneum is deperitonealized by traction with Allis clamps, and the retropubic space of Retzuis is developed. A plane of dissection is established between the anterior pelvic peritoneum and the bladder dome muscularis using the electrosurgical unit (ESU) or Metzenbaum scissor. The deperitonealizing direction is ventral to dorsal and lateral to medial toward the uterus until the pubovesicocervical fascia is reached.

The bladder is sharply mobilized ventrocaudally to expose the proximal $2-3 \mathrm{~cm}$ of vagina and vesicovaginal space developed.

The ureter is extricated from within the bladder pillar bilaterally by developing the ureteral tunnel using a rightangle clamp and suture ligating or advanced bipolar devices.

The hysterectomy is completed in a retrograde fashion by first making an anterior colpotomy. Bimanual vaginal examination or placement of a sponge stick into anterior vaginal fornix will facilitate selecting the proper colpotomy site of vagina. Anterior colpotomy expand transversely to the lateral vagina. Allis clamps are used on the cutting edge 

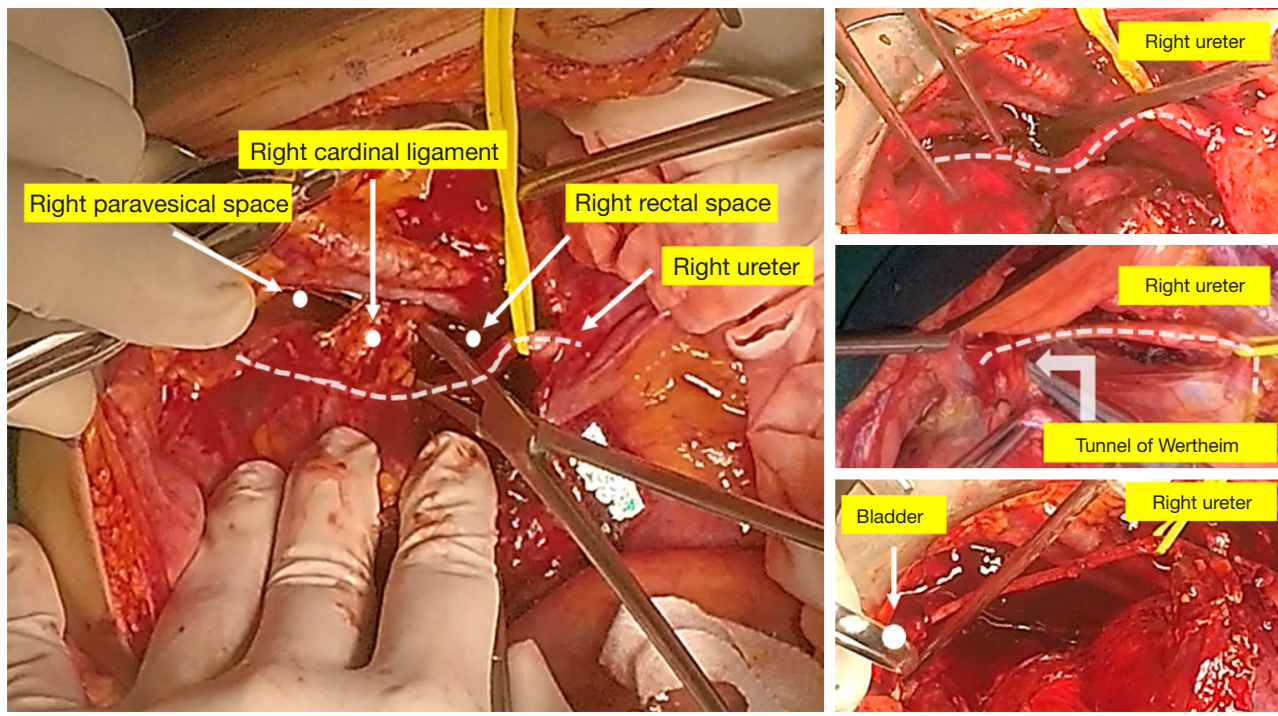

Figure 3 The cardinal ligament is identifying by developing the pararectal and paravesicle spaces. Mobilize the ureter to see the path to the bladder. After developing the Tunnel of Wertheim, place the ureter anterolaterally and cut the Tunnel of Wertheim. Then we can see the ureter entering the trigone of the bladder.
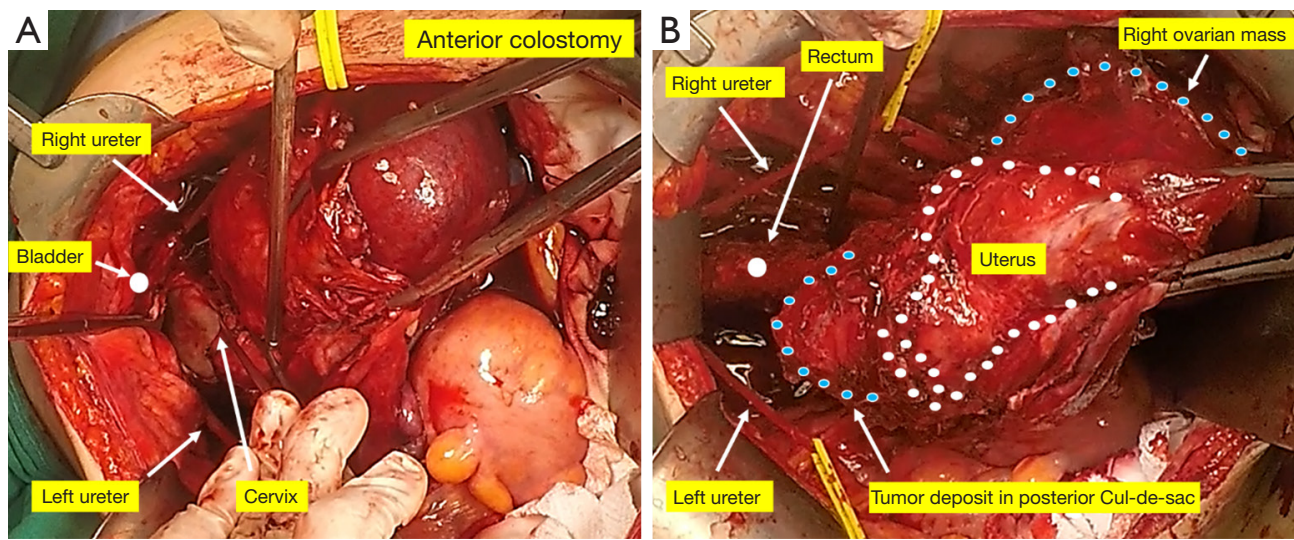

Figure 4 The hysterectomy is completed in a retrograde fashion by first making an anterior colpotomy. (A) Anterior colpotomy expose the vaginal lumen. Allis clamps are used to clamp on the cutting edge of anterior vagina. (B) Using the Allis clamp, traction the cervix and uterus helps dissection. The posterior vaginal wall is also incised and the rectovaginal space developed caudally along the rectum until it reached to the lowest $2-3 \mathrm{~cm}$ of margin from tumor deposit.

of vagina in sequence. Tractions of the cervix and uterus helps dissection. The posterior vaginal wall is incised with the ESU and the rectovaginal space is developed caudally along the rectum until it reaches to the lowest safety margin $(2-3 \mathrm{~cm})$ from tumor (Figure 4).

The retrograde approach is continued by retracting the posterior cul-de-sac upward, exposing the remaining cardinal ligament, the uterosacral ligaments, and the rectal pillars. These are sequentially divided using clamp and suture ligatures or advanced bipolar devices.

The type I or type II radical oophorectomy can be selected depending on the extent of tumor involvement with the anterior rectal wall or sigmoid colon.

\section{Type I modification}

With only superficial involvement of tumor to the rectum, the tumor and peritoneum can be sharply dissected from 

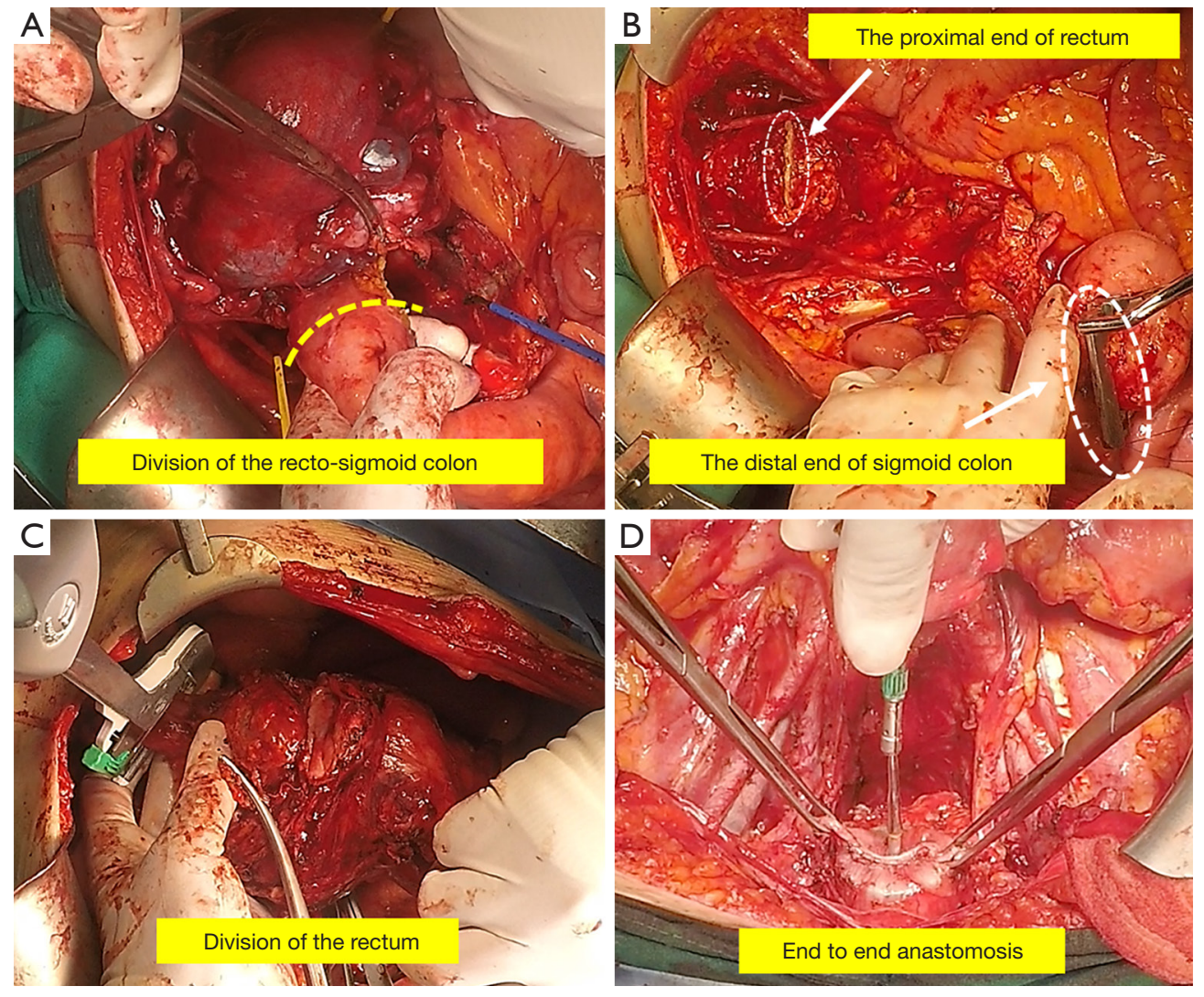

Figure 5 The recto-sigmoid colon is divided. (A) The rectum is dissected at 2-3 cm from at the lowest of tumor for safety margin; (B) the rectum is divided at $2-3 \mathrm{~cm}$ from at the lowest of tumor by using TA stapler and a proximal bowel clamp; (C) the pelvic mass removed en bloc with uterus and rectosigmoid colon; (D) the anvil shaft inserted into the cartidge shaft of the main CEEA instrument, until it sounds click.

the anterior surface of the rectum and the sigmoid colon. If the tumor penetrates into the muscularis of the rectum within limited extent $(\leq 2 \mathrm{~cm})$, “wedge-shaped” segment of rectal wall can be excised and repaired by using a fine monofilament suture in interrupted inverting stitches, incorporating minimal mucosa, placed perpendicular to the long axis of the bowel or by using stapling technique.

\section{Type II modification}

The type II modification of radical oophorectomy is indicated for disease which has extensive involvement of the posterior cul-de-sac and rectosigmoid colon.

Division of the proximal sigmoid colon can be performed whenever it is most convenient during the operation (Figure $5 A$ ), once it has been determined that bowel resection is necessary to achieve an optimal cytoreduction after surgery. The sigmoid colon is divided $2-3 \mathrm{~cm}$ above the most proximal of tumor. Depending on the planned anastomosis, a variety of methods can be used to divide the sigmoid colon. The GIA ${ }^{\mathrm{TM}}$ (Medtronic, Inc., Minneapolis, MN, USA) stapling device is the most expedient and has advantage of placing double rows of staples on either side of the divided bowel. Or using a purse-string clamp, circular end-to-end (CEEA) anvil is inserted and purse-string suture tied within the notch on the anvil shaft. It is important that the bowel wall be cleared of fat for a $2-3 \mathrm{~cm}$ from distance to the planned anastomotic site.

The deep dissection proceeds from the pararectal spaces posteromedially toward the entrance of the presacral space just caudal to the sacral promontory. Depending on the surgeon's preference, one can choose to dissect either TME or CRD. If the inferior mesenteric artery and vein are sacrificed, be careful to preserve the left colic artery with its blood supply to the descending colon. The posterior pelvis is further mobilized by developing the presacral space 
caudally to the level of pelvic floor musculature.

The posterior cul-de-sac tumor mass is mobilized by exposing the rectovaginal space and dissecting to the point at which the tumor directly invades the bowel wall. The bowel wall should be cleared of fat and any remaining mesorectal attachments. The rectum is then divided at $2-3 \mathrm{~cm}$ from the lowest of tumor by using $\mathrm{TA}^{\mathrm{TM}}$ (Medtronic, Inc., Minneapolis, MN, USA) stapler and a proximal bowel clamp (Figure 5B).

The central pelvic mass is removed en bloc with the rectosigmoid colon (Figure 5C).

The divided end of the proximal colon is brought down to the rectal stump to confirm that anastomosis will be tension-free. If it needs additional mobilization of the sigmoid and descending colon, several maneuvers can be applied.

Intestinal continuity can be reestablished by a variety of methods using either automated stapling devices or hand-sewn technique. The transanal double-stapling technique using the CEEA is a safe and efficient method of reestablishing intestinal continuity. In general, at least a 28 or $31 \mathrm{~mm}$ stapler should be used. Blunt rectal sizing instruments are gently passed into the distal bowel segment. The main CEEA instrument is lubricated and inserted into the rectum transanally (with closed trocar status), grasp the handle of the main CEEA instrument and place the cartridge head on the staple line of the rectal stump. The wing nut is rotated counterclockwise until the trocar pierces the closed rectal stump adjacent to or through the staple line. If there is concern that the colonic tissue between the circular and linear rows of staples may become ischemic or leakage, the trocar can be directed $2 \mathrm{~cm}$ anterior or posterior to the linear row of staple, avoiding a staple-tostaple intersection in the anastomotic ring.

The anvil shaft inserted into the cartridge shaft of the main CEEA instrument until it sounds click (Figure 5D).

The wing nut is rotated clockwise, keeping the bowel on stretch and pressure, until the color bar indicator on the handle of the main CEEA instrument is in the proper position indicating adequate compression of the anastomotic site.

Hold several seconds for reforming of the tissue of the anastomotic site and stapler fired by squeezing the handles together as far as they go until it sounds crunch. The handles are released and the wing nut rotated counterclockwise $180^{\circ}-360^{\circ}$ (no more than $360^{\circ}$ ) to release the anastomotic site. The handpiece of the main CEEA instrument is removed carefully to avoid disrupting the staple line during the removal.

Some surgeons recommend manually hand-sewing with interrupted stitches over the staples to reinforce the anastomotic site. The security of the anastomosis is confirmed by several means. First, the resection rings around the cartridge shaft should be inspected to ensure two complete donuts of colonic tissue. Second, the "bubble test" or "water test" is performed by filling the pelvis with normal saline or water and obstructing the proximal colon manually prior to insufflating the rectum with 200-300 cc of air through a rectal tube or Asepto syringe. The presence of air bubbles indicates an anastomotic leak. It should be repaired by oversewing the defect with interrupted stitches of 3-0 delayed absorbable or silk suture.

\section{Conclusions}

In summary, primary debulking procedures with the aim of maximal cytoreduction is strongly advocated in patients with advanced EOC. The techniques of en bloc resection of the rectosigmoid colon with the pelvic tumor mass may assist complete removal of all visible pelvic tumor, thereby improving survival benefits that optimal cytoreduction allows. Literatures reported to date on en bloc pelvic resection with rectosigmoid colectomy in advanced EOC implies that the benefits of the surgical procedures outweigh the potential morbidity issues with feasibility and safety. For achieving complete resection of pelvic disease, en bloc pelvic resection with rectosigmoid colectomy may be considered as a highly effective and safe surgical technique.

\section{Acknowledgments}

The authors are immensely grateful to Drs. Sang-Yoon Park and Jae-Weon Kim for sharing their pearls of wisdom during the course of this research and for providing comments on an earlier version of the manuscript. Any errors of this manuscript are our own and should not tarnish the reputation of them.

Funding: This work was supported by the National Research Foundation of Korea Grant, funded by the government of Republic of Korea (Ministry of Science and Information Technology) [2019R1F1A1063567].

\section{Footnote}

Provenance and Peer Review: This article was commissioned by the Guest Editors (Sang Yoon Park, Jae Weon Kim) 
for the series "Ultra-Radical Surgery in Ovarian Cancer: Surgical Techniques for Gynecologic Oncologist" published in Gland Surgery. The article was sent for external peer review organized by the Guest Editors and the editorial office.

Conflicts of Interest: All authors have completed the ICMJE uniform disclosure form (available at http:// dx.doi.org/10.21037/gs-19-540). The series "UltraRadical Surgery in Ovarian Cancer: Surgical Techniques for Gynecologic Oncologist" was commissioned by the editorial office without any funding or sponsorship. The authors have no other conflicts of interest to declare.

Ethical Statement: The authors are accountable for all aspects of the work in ensuring that questions related to the accuracy or integrity of any part of the work are appropriately investigated and resolved.

Open Access Statement: This is an Open Access article distributed in accordance with the Creative Commons Attribution-NonCommercial-NoDerivs 4.0 International License (CC BY-NC-ND 4.0), which permits the noncommercial replication and distribution of the article with the strict proviso that no changes or edits are made and the original work is properly cited (including links to both the formal publication through the relevant DOI and the license). See: https://creativecommons.org/licenses/by-nc-nd/4.0/.

\section{References}

1. Chang SJ, Bristow RE, Chi DS, et al. Role of aggressive surgical cytoreduction in advanced ovarian cancer. J Gynecol Oncol 2015;26:336-42.

2. Chang SJ, Hodeib M, Chang J, et al. Survival impact of complete cytoreduction to no gross residual disease for advanced-stage ovarian cancer: a meta-analysis. Gynecol Oncol 2013;130:493-8.

3. Griffiths CT. Surgical resection of tumor bulk in the primary treatment of ovarian carcinoma. Natl Cancer Inst Monogr 1975;42:101-4.

4. Hacker NF, Berek JS, Lagasse LD, et al. Primary cytoreductive surgery for epithelial ovarian cancer. Obstet Gynecol1983;61:413-20.

5. Conte PF, Sertoli MR, Bruzzone M, et al. Cisplatin, methotrexate, and 5-fluorouracil combination chemotherapy for advanced ovarian cancer. Gynecol Oncol 1985;20:290-7.
6. Neijt JP, ten Bokkel Huinink WW, van der Burg ME, et al. Randomized trial comparing two combination chemotherapy regimens (CHAP-5 v CP) in advanced ovarian carcinoma. J Clin Oncol 1987;5:1157-68.

7. Piver MS, Lele SB, Marchetti DL, et al. The impact of aggressive debulking surgery and cisplatin-based chemotherapy on progression-free survival in stage III and IV ovarian carcinoma. J Clin Oncol 1988;6:983-9.

8. Eisenkop SM, Spirtos NM, Montag TW, et al. The impact of subspecialty training on the management of advanced ovarian cancer. Gynecol Oncol 1992;47:203-9.

9. Michel G, De Iaco P, Castaigne D, et al. Extensive cytoreductive surgery in advanced ovarian carcinoma. Eur J Gynaecol Oncol 1997;18:9-15.

10. Bristow RE, Tomacruz RS, Armstrong DK, et al. Survival effect of maximal cytoreductive surgery for advanced ovarian carcinoma during the platinum era: a metaanalysis. J Clin Oncol 2002;20:1248-59.

11. Bertelsen K. Tumor reduction surgery and long-term survival in advanced ovarian cancer: a DACOVA study. Gynecol Oncol 1990;38:203-9.

12. Hoffman MS, Griffin D, Tebes S, et al. Sites of bowel resected to achieve optimal ovarian cancer cytoreduction: Implications regarding surgical management. Am J Obstet Gynecol 2005;193:582-6.

13. Gillette-Cloven N, Burger RA, Monk BJ, et al. Bowel resection at the time of primary cytoreduction for epithelial ovarian cancer. J Am Coll Surg 2001;193:626-32.

14. Baik SH, Kim NK, Lee YC, et al. Prognostic significance of circumferential resection margin following total mesorectal excision and adjuvant chemoradiotherapy in patients with rectal cancer. Ann Surg Oncol 2007;14:462-9.

15. Wibe A, Rendedal PR, Svensson E, et al. Prognostic significance of the circumferential resection margin following total mesorectal excision for rectal cancer. $\mathrm{Br} \mathrm{J}$ Surg 2002;89:327-34.

16. Son JH, Kim J, Shim J, et al. Comparison of posterior rectal dissection techniques during rectosigmoid colon resection as part of cytoreductive surgery in patients with epithelial ovarian cancer: Close rectal dissection versus total mesorectal excision. Gynecol Oncol 2019;153:362-7.

17. Kim M, Suh DH, Park JY, et al. Survival impact of low anterior resection in patients with epithelial ovarian cancer grossly confined to the pelvic cavity: a Korean multicenter study. J Gynecol Oncol 2018;29:e60.

18. Hudson CN. A radical operation for fixed ovarian tumours. J Obstet Gynaecol Br Commonw 
1968;75:1155-60.

19. Hudson CN, Chir M. Surgical treatment of ovarian cancer. Gynecol Oncol 1973;1:370-8.

20. Clayton RD, Obermair A, Hammond IG, et al. The Western Australian Experience of the Use of en Bloc Resection of Ovarian Cancer with Concomitant Rectosigmoid Colectomy. Gynecol Oncol 2002;84:53-7.

21. Berek JS, Hacker NF, Lagasse LD. Rectosigmoid colectomy and reanastomosis to facilitate resection of primary and recurrent gynecologic cancer. Obstet Gynecol 1984;64:715-20.

22. Soper JT, Couchman G, Berchuck A, et al. The role of partial sigmoid colectomy for debulking epithelial ovarian carcinoma. Gynecol Oncol 1991;41:239-44.

23. Obermair A, Hagenauer S, Tamandl D, et al. Safety and efficacy of low anterior en bloc resection as part of cytoreductive surgery for patients with ovarian cancer. Gynecol Oncol 2001;83:115-20.

24. Bridges JE, Leung Y, Hammond IG, et al. En bloc resection of epithelial ovarian tumors with concomitant rectosigmoid colectomy: the KEMH experience. Int J Gynecol Cancer 1993;3:199-202.

25. Barnes W, Johnson J, Waggoner S, et al. Reverse hysterocolposigmoidectomy (RHCS) for resection of panpelvic tumors. Gynecol Oncol 1991;42:151-5.

26. Sugarbaker PH. Complete parietal and visceral peritonectomy of the pelvis for advanced primary and recurrent ovarian cancer. Cancer Treat Res 1996;81:75-87.

27. Sainz de la Cuesta R, Goodman A, Halverson SS. En bloc pelvic peritoneal resection of the intraperitoneal pelvic viscera in patients with advanced epithelial ovarian cancer. Cancer J Sci Am 1996;2:152-7.

28. Eisenkop SM, Nalick RH, Teng NN. Modified posterior exenteration for ovarian cancer. Obstet Gynecol 1991;78:879-85.

29. Sainz de la Cuesta R, Goodman A, Halverson S. En bloc pelvic peritoneal resection of the intraperitoneal pelvic viscera in patients with advanced epithelial ovarian cancer. Cancer J Sci Am 1996;2:152-7.

30. Park JY, Seo SS, Kang S, et al. The benefits of low anterior en bloc resection as part of cytoreductive surgery for advanced primary and recurrent epithelial ovarian cancer patients outweigh morbidity concerns. Gynecol Oncol 2006;103:977-84.

31. Bristow RE, del Carmen MG, Kaufman HS, et al. Radical oophorectomy with primary stapled colorectal anastomosis for resection of locally advanced epithelial ovarian cancer. J Am Coll Surg 2003;197:565-74.
32. Chi DS, Franklin CC, Levine DA, et al. Improved optimal cytoreduction rates for stages IIIC and IV epithelial ovarian, fallopian tube, and primary peritoneal cancer: a change in surgical approach. Gynecol Oncol 2004;94:650-4.

33. Chi DS, Eisenhauer EL, Zivanovic O, et al. Improved progression-free and overall survival in advanced ovarian cancer as a result of a change in surgical paradigm. Gynecol Oncol 2009;114:26-31.

34. Bretagnol F, Alves A, Ricci A, et al. Rectal cancer surgery without mechanical bowel preparation. Br J Surg 2007;94:1266-71.

35. Cao F, Li J, Li F. Mechanical bowel preparation for elective colorectal surgery: updated systematic review and meta-analysis. Int J Colorectal Dis 2012;27:803-10.

36. Zhu QD, Zhang QY, Zeng QQ, et al. Efficacy of mechanical bowel preparation with polyethylene glycol in prevention of postoperative complications in elective colorectal surgery: a meta-analysis. Int J Colorectal Dis 2010;25:267-75.

37. Dolejs SC, Guzman MJ, Fajardo AD, et al. Bowel Preparation Is Associated with Reduced Morbidity in Elderly Patients Undergoing Elective Colectomy. J Gastrointest Surg 2017;21:372-9.

38. Mahajna A, Krausz M, Rosin D, et al. Bowel preparation is associated with spillage of bowel contents in colorectal surgery. Dis Colon Rectum 2005;48:1626-31.

39. Atkinson SJ, Swenson BR, Hanseman DJ, et al. In the Absence of a Mechanical Bowel Prep, Does the Addition of Pre-Operative Oral Antibiotics to Parental Antibiotics Decrease the Incidence of Surgical Site Infection after Elective Segmental Colectomy? Surg Infect (Larchmt) 2015;16:728-32.

40. Gustafsson UO, Scott MJ, Hubner M, et al. Guidelines for Perioperative Care in Elective Colorectal Surgery: Enhanced Recovery After Surgery (ERAS(®)) Society Recommendations: 2018. World J Surg 2019;43:659-95.

41. Gustafsson UO, Scott MJ, Schwenk W, et al. Guidelines for perioperative care in elective colonic surgery: Enhanced Recovery After Surgery (ERAS®) Society recommendations. Clin Nutr 2012;31:783-800.

42. Nelson RL, Glenny AM, Song F. Antimicrobial prophylaxis for colorectal surgery. Cochrane Database Syst Rev 2009;(1):CD001181.

43. Noh JJ, Kim MS, Lee YY. The implementation of enhanced recovery after surgery protocols in ovarian malignancy surgery. Gland Surg 2021;10:1182-94.

44. Hannemann P, Lassen K, Hausel J, et al. Patterns in 
current anaesthesiological peri-operative practice for colonic resections: a survey in five northern-European countries. Acta Anaesthesiol Scand 2006;50:1152-60.

45. Lewis SJ, Egger M, Sylvester PA, et al. Early enteral feeding versus "nil by mouth" after gastrointestinal surgery: systematic review and meta-analysis of controlled trials. BMJ 2001;323:773-6.

46. Han-Geurts IJ, Hop WC, Kok NF, et al. Randomized clinical trial of the impact of early enteral feeding on postoperative ileus and recovery. Br J Surg 2007;94:555-61.

47. Midura EF, Hanseman D, Davis BR, et al. Risk factors and consequences of anastomotic leak after colectomy: a national analysis. Dis Colon Rectum 2015;58:333-8.

48. Venara A, Neunlist M, Slim K, et al. Postoperative ileus: Pathophysiology, incidence, and prevention. J Visc Surg 2016;153:439-46.

49. Lee MJ, Vaughan-Shaw P, Vimalachandran D. A systematic review and meta-analysis of baseline risk factors for the development of postoperative ileus in patients undergoing gastrointestinal surgery. Ann R Coll Surg Engl 2020;102:194-203.

50. Chapuis PH, Bokey L, Keshava A, et al. Risk Factors for Prolonged Ileus After Resection of Colorectal Cancer: An Observational Study of 2400 Consecutive Patients. Ann Surg 2013;257:909-15.

51. Moghadamyeghaneh Z, Hwang GS, Hanna MH, et al. Risk factors for prolonged ileus following colon surgery.

Cite this article as: Kim MS, Noh JJ, Lee YY. En bloc pelvic resection of ovarian cancer with rectosigmoid colectomy: a literature review. Gland Surg 2021;10(3):1195-1206. doi: 10.21037 /gs-19-540
Surg Endosc 2016;30:603-9.

52. Svatek RS, Fisher MB, Williams MB, et al. Age and Body Mass Index Are Independent Risk Factors for the Development of Postoperative Paralytic Ileus After Radical Cystectomy. Urology 2010;76:1419-24.

53. Vather R, Josephson R, Jaung R, et al. Development of a risk stratification system for the occurrence of prolonged postoperative ileus after colorectal surgery: A prospective risk factor analysis. Surgery 2015;157:764-73.

54. Artinyan A, Nunoo-Mensah JW, Balasubramaniam S, et al. Prolonged Postoperative Ileus-Definition, Risk Factors, and Predictors after Surgery. World J Surg 2008;32:1495-500.

55. Millan M, Biondo S, Fraccalvieri D, et al. Risk factors for prolonged postoperative ileus after colorectal cancer surgery. World J Surg 2012;36:179-85.

56. Vather R, Trivedi S, Bissett I. Defining Postoperative Ileus: Results of a Systematic Review and Global Survey. J Gastrointest Surg 2013;17:962-72.

57. Flores-Funes D, Campillo-Soto Á, Pellicer-Franco E, et al. The use of coffee, chewing-gum and gastrograffin in the management of postoperative ileus: A review of current evidence. Cir Esp 2016;94:495-501.

58. Benedetti-Panici P, Maneschi F, Scambia G, et al. The pelvic retroperitoneal approach in the treatment of advanced ovarian carcinoma. Obstet Gynecol1996;87:532-8. 